\title{
Evaluation of the Effect of Perceived Social Support on Promoting Self- Care Behaviors of Heart Failure Patients Referred to The Cardiovascular Research Center of Isfahan
}

\author{
Gholam Hassan Khaledi ${ }^{1}$; Ahmad Ali Eslami ${ }^{1{ }^{1}}$; Hamidreza Rooh Afza ${ }^{2}$; Firoozeh Mostafavi ${ }^{1}$; \\ Hassanzadeh Akbar ${ }^{3}$ \\ ${ }^{1}$ Department of Health Education and Promotion, Faculty of Health, Isfahan University of Medical Sciences, Isfahan, IR Iran \\ ${ }_{3}^{2}$ Cardiovascular Research Center of Isfahan, Isfahan University of Medical Sciences, Isfahan, IR Iran \\ ${ }^{3}$ Department of Statistics and Epidemiology, Faculty of Health, Isfahan University of Medical Sciences, Isfahan, IR Iran \\ ${ }^{*}$ Corresponding Author: Ahmad Ali Eslami, Department of Health Education and Promotion, Faculty of Health, Isfahan University of Medical Sciences, Isfahan, IR Iran. E-mail: esla- \\ miaa@gmail.com
}

Received: August 20, 2014; Revised: February 12, 2015; Accepted: March 13, 2015

\begin{abstract}
Background: Self-care is one of the most important aspects of treatment in patients with heart failure and ranks among the most important coping strategies against the events and stresses of life. Perceived social support plays an important role in performing self-care behaviors in these patients.

Objectives: This study was conducted to evaluate the effect of perceived social support on promoting self-care behaviors among heart failure patients.

Patients and Methods: This educational intervention with a randomized control group was performed on 64 heart failure patients referred to The Cardiovascular Research Center of Isfahan. The study population was divided randomly into two groups of intervention and control. The indicators of self-care behavior and perceived social support (before, immediately after, and 2 months after the intervention) were completed by the two groups. The intervention group received educational interventions in 120-minute sessions once a week for 4 weeks. SPSS software (version 20) was used for data analysis in addition to methods of descriptive and inferential statistics.

Results: Based on the obtained results, educational intervention was effective in the improvement of perceived social support among our heart failure patients. The results also showed that an increase in perceived social support significantly promoted self-care behaviors in the case group after the intervention compared with the control group $(\mathrm{P}<0.001)$.

Conclusions: Perceived social support played an important role in improving the performance of self-care behaviors in our heart failure patients. Given the strengths of the present study, these findings can be considered in future research in this domain.
\end{abstract}

Keywords: Social Support; Self Care; Heart Failure; Patients

\section{Background}

Cardiovascular diseases currently are one of the most common chronic diseases in elderly and middle-aged people and also the leading cause of hospitalization in older people (1). Heart failure is the most common disease among the different types of cardiovascular diseases (2). Cardiovascular diseases mostly occur in middle ages, when the individual is needed by both family and government. In our country, the low mean age at the onset of cardiovascular diseases by comparison with many other countries has it many negative corollaries such as socioeconomic costs. On a global scale, cardiovascular diseases are regarded as a complicated health problem (3). New medical and surgical treatment modalities have increased the longevity of cardiovascular disease patients and, thus, increased the overall incidence rate of heart failure (4). From a clinical point of view, heart failure can be deemed a pathophysiological state. In this disease, cardiac dysfunction renders it unable to pump sufficient blood to tissues, unless there is an abnormal rise in the ventricular diastolic volume (5). According to the latest statistics by the World Health Organization, more than $80 \%$ of cardiovascular diseases occur in low-and moderate-income countries. The statistics also indicate that almost half of cardiovascular diseases in these countries occur in the age group of below 70 years and about a quarter of cases occur in the age group of below 60 years. The interesting point is that until the age of 40 years, the risk of heart diseases in men is twice that in women. This ratio decreases with an increase in age and from age of 55 years onwards, susceptibility to this disease is almost identical in men and women. In our country according to the latest reports, cardiovascular diseases are account-

Copyright ( 2015, Iranian Red Crescent Medical Journal. This is an open-access article distributed under the terms of the Creative Commons Attribution-NonCommercial 4.0 International License (http://creativecommons.org/licenses/by-nc/4.0/) which permits copy and redistribute the material just in noncommercial usages, provided the original work is properly cited. 
able for more than $38 \%$ (or about $50 \%$ ) of mortalities and morbidities. Predictions suggest that by 2020 cardiovascular diseases will have been the cause of more than $75 \%$ of mortalities and morbidities at global level $(6,7)$. Several studies have shown that contextual factors have an important role in the incidence of heart failure (8-10). For example, a study conducted at the University of California indicated that gender, age, and hypertension are associated with heart failure (11). A study in Switzerland demonstrated an association between age, gender, and blood pressure and the incidence of diabetes and heart failure (11). Some studies have pointed to the role of awareness in the incidence of heart failure $(9,11)$. One such study in the US underscored the relationship between smoking and reduced physical activity and heart failure (12). Several studies have pointed out the relationship between perceived social support and heart failure $(9,11,12)$. Most cardiovascular risk factors are related to awareness and behavior. Therefore, one of the most important therapeutic measures in these patients is performing self-care behaviors. (12) According to Orem's definition, self-care is a regulatory function in humans based on the individual's ability to perform self-care practices on themselves (13, 14). Self-care is defined as a coping strategy against life events and stresses triggered by an increase in the process of aging and dependence. With respect to cardiovascular diseases, self-care comprises special activities to reduce and relieve symptoms and maintain and promote health in patients. Self-care is, therefore, one of the most significant aspects of the treatment of patients with heart failure (15) and includes activities such as daily weight measurement; contacting a physician when observing a swelling on the foot, ankle, legs, and abdomen; being cautious about the amount of daily urine; regular consumption of medicines as directed by a physician; and refraining from drinking too much fluids. It is required that non-medical self-care training be a part of the daily management of patients with heart failure in hospitals and emergency environments $(16,17)$. There are many factors which exert an influence on the performance of self-care behaviors in heart failure patients such as awareness, education level, number of hospitalization, and perceived social support $(18,19)$. Social support is one of the most significant factors influencing mental condition and treatment follow-up in patients with chronic diseases, especially heart failure (20). Due to the debilitating nature of this disease, the plan for the care of these patients should take into account all aspects of life, including social support (21). Social support can be defined as the rate of receiving the affection, attention, and assistance of family members, relatives, and friends (22). Perceived social support means the individual's perception of the availability of the support in conjunction with its adequacy and quality. The mental aspect of social support can be an effective assistance to the person in need of mental help to cope with stresses of life because such an individual in all moments of life has this clear notion in mind that there are always people available to lend a helping hand at the time of helplessness. Consequently, the individual will feel strengthened when faced with adversity (23). Moreover, social support has a great impact on the level of individual's performance when dealing with complex problems $(24,25)$. According to Friedmann et al. (26), social support from family and friends, for instance in the shape of receiving a shelter to live in, can be a facilitating factor in the treatment and improvement of related health outcomes. The authors state that receiving appropriate social support from the family accelerates the recovery period, encourages self-care behaviors, and reduces the risk of relapse in patients. There is evidence that perceived social protection can lessen the physiological adverse effects of disease and clearly augment the individual's performance. Life motivation and self-care behaviors are highly correlated and can contribute to the prevention of symptom development, reduction in the recovery period, and prevention of the recurrence of the illness in heart failure patients $(24,25,27)$.

\section{Objectives}

Given the relationship between perceived social support and self-care performance in heart failure patients, the present study sought to assess the effect of perceived social support on promoting self-care behavior in patients with heart failure referred to The Cardiovascular Research Center of Isfahan in 2012-2013.

\section{Patients and Methods}

This quasi-experimental research was conducted in two stages between 2012 and 2013 on 64 patients with heart failure divided into two groups of intervention and control. The study population included all patients with heart failure referred to The Cardiovascular Research Center of Isfahan. The inclusion criteria consisted of having a record at the center, residence in Isfahan, consent to participation, and lack of involvement in other similar programs. The diagnosis of heart failure was based on the following indicators: 1) positive echocardiogram and 2) having a history of heart failure for at least 6 months. All the 64 patients, who were initially recruited, remained in the study. Normal assumption was checked using the Kolmogorov-Smirnov test. Sample size was determined via Equation 1:

$$
n=\frac{\left(Z_{1}+Z_{2}\right)^{2}(2 S)^{2}}{(d)^{2}}
$$

$\mathrm{n}=$ Sample size

$\mathrm{z}_{1}=$ Confidence factor test $0.95=1.96$

$\mathrm{z}_{2}=$ Power factor test $0.80=0.84$

$\mathrm{s}=$ Standard deviation pool

$\mathrm{d}=$ Smallest difference of interest between the two groups $=0.7 \mathrm{~s}$ 
Khaledi GH et al.

\subsection{Tools}

The tools employed in the present study included questionnaires of perceived social support and self-care behaviors. The perceived social support questionnaire was comprised of 12 questions, graded based on a 7-point Likert scale. For example, the first question of the questionnaire was: "There is always a particular person by my side when I need them." (totally disagree: 1, disagree: 2 , somewhat disagree: 3 , have no comment: 4 , somewhat agree: 5 , agree: 6 , and totally agree: 7 ). Obtainable points in the perceived social support questionnaire were variable between 12 and 84, with higher ratings denoting better conditions in perceived social support. Zimet et al. (28) reported the alpha reliability coefficient of this tool in the range of 0.85 to 0.91 and 0.72 to 0.85 via the retest method. In Iran, this questionnaire has been previously used by Davari et al. (29), who confirmed the reliability and validity of this questionnaire with a correlation coefficient of 0.895 according to test-retest and Cronbach's alpha coefficient of $87 \%$. The self-care behavior questionnaire of Europe contains 15 questions, graded on the basis of a 5-point Likert scale. For example, the first question of the questionnaire is: "I weigh myself every day." (never: 1, rarely: 2 , sometimes: 3 , often: 4 , and always: 5 ). The obtainable points in the self-care behavior questionnaire were variable between 15 to 75 , with higher scores indicating better conditions of self-care behavior. Another study reported a correlation coefficient of 0.94 by calculating Cronbach's alpha of 0.8 . The questionnaire has also been used in Iran by Shojahfard et al. (30), who determined Cronbach's alpha coefficient and Pearson correlation coefficient of 0.86 and 0.94 , respectively.

\subsection{Methods}

A preliminary meeting was held for the study subjects and their closest companions in order to perform the pre-test and meet the research objectives, research process, and also the methods of meetings and their planning. Written informed consent was obtained from all the participants. The patients were asked not to take part in other consulting and training programs during the implementation of this study and to continue their daily routine without any change. Demographic information such as age, gender, and level of education was collected from all the participants, and they completed the perceived social support and self-care behavior questionnaires. Then, the subjects (and their companions) were categorized randomly to two groups of intervention and control via the randomized allocation method. The intervention group was divided into 3 subgroups: one group of 10 and two groups of 11 persons. The closest person to the patient participated in 4 sessions of the scheduled treatment interventions, one day per week. The meetings were held in the classrooms of the heart center. The patients' companions were invited to the center to attend the intervention sessions on a regular basis during the interventions based on a list compiled by the secretary of the heart center. The therapy sessions were held 4 times, one session per week and each session lasted for 2 hours in a group. The educational contents of the meeting encompassed heart failure, self-care behaviors and their importance, and perceived social support and its significance. After the 4 th session, the subjects were asked to complete the above questionnaires once more. For the purposes of follow-up, the participants were invited to return to the heart center 2 months after the completion of training to fill in the questionnaires. The patients were asked to contact the researcher in the event of problems and the need for more information during this 2-month period. Finally, the statistical analyses were performed using the chi-square test, Mann-Whitney test, independent t-test, analysis of variance (ANOVA) with repeated observations, and analysis of covariance. Finally, the studied variables were analyzed. (The data extraction center was a governmental organization named Isfahan Cardiovascular Research Institute.)

\section{Results}

The study population had a mean age of $54.4 \pm 7.8$ years: $54.8 \pm 7.8$ years in the intervention group and $54.3 \pm 8.1$ years in the control group. All the participants were married. In terms of demographics (i.e. age, gender, marital status, and level of education), there were no significant differences between the two groups $(P=0.802)$. In the evaluation of the status of perceived social support based on the obtained data from both groups, the independent t-test showed no significant difference between the two groups before the intervention as regards the mean score for perceived social support $(\mathrm{P}=0.53)$ (Table 1 ).

However, as is demonstrated by Table 2, after performing the educational intervention program, the mean score for perceived social support in a 2-stage assessment (immediately and 2 months after the intervention) was significantly higher in the intervention group than in the control group $(\mathrm{P}<0.001)$. In investigating the status of perceived social support in the control group, the paired t-test showed that its mean score(before the intervention and 2 months after the intervention) had no significant difference $(\mathrm{P}=0.36)$. On the other hand, the ANOVA with repeated observations showed that in the intervention group, the mean scores for perceived social support in three stages (i.e. before, immediately after, and 2 months after the intervention ) had significant differences with one another $(\mathrm{P}<0.001)$. The least significant difference post-hoc test indicated that the mean score for perceived social support of the patients increased significantly immediately after the intervention compared with that before the intervention $(\mathrm{P}<0.001)$. Moreover, the mean score for perceived social support showed a reduction 2 months after the intervention compared with that immediately after the intervention $(\mathrm{P}=0.001)$, whereas it was significantly higher 2 months after the intervention than that before the intervention $(\mathrm{P}<0.001)$ (Table 2$)$. 
In checking-out the status of self-care behaviors based on the obtained data from the two groups, the independent t-test showed no significant difference in the mean score for self-care behaviors between the two groups before the intervention $(\mathrm{P}=0.803)$. However, according to Table 3, after performing the educational intervention program, the mean score for self-care behaviors assessed in two stages (i.e. immediately and 2 months after the intervention) in the intervention group was significantly higher than that in the control group $(\mathrm{P}<0.001)$. In the evaluation of the status of selfcare behaviors in the control group, the paired t-test demonstrated no significant differences in the mean scores for self-care behaviors before and 2 months af- ter the intervention $(\mathrm{P}=0.28)$. Likewise, the repeated ANOVA test with repeated measurements revealed no significant difference in the case group regarding the mean scores for self-care behaviors in the three stages of the study $(\mathrm{P}<0.001)$. Meanwhile, the least significant difference post-hoc test showed that the mean score for self-care behaviors in the patients rose significantly immediately after the intervention compared with that before the intervention $(\mathrm{P}<0.001)$ and declined 2 months after the intervention compared with that immediately after the intervention $(P=0.01)$. However, the mean score 2 months after the intervention was significantly higher than that before the intervention $(\mathrm{P}<0.001)$ (Table 3$)$.

\begin{tabular}{|c|c|c|c|}
\hline Variable & Intervention Group & Control Group & Test \\
\hline Gender & & & $\chi^{2}=1.72 ;$ P value $=0.189$, Chi square \\
\hline Male & $22(68.8)$ & $22(68.8)$ & \\
\hline Female & $10(31.2)$ & $10(31.2)$ & \\
\hline Marital status & & & P Value $=0.243$, Fisher exact test \\
\hline Single & $0(0)$ & $0(0)$ & \\
\hline Married & $32(100)$ & $32(100)$ & \\
\hline Education level & & & $\mathrm{z}=0.236 ;$ P Value $=0.84$, Mann-Whitney test \\
\hline Under Diploma & $11(34.4)$ & $4(12.5)$ & \\
\hline Diploma & $8(25.8)$ & $13(40.6)$ & \\
\hline Technician & $10(31.2)$ & $11(34.4)$ & \\
\hline BSc & $2(6.2)$ & $4(12.5)$ & \\
\hline MSc & $0(0)$ & $1(3.2)$ & \\
\hline Age, $y$ & $54.8(7.8)$ & $54.3(8.1)$ & $\mathrm{t}=0.14$ (Independent $\mathrm{t}$-test $) ; \mathrm{P}$ value $=0.88$ \\
\hline
\end{tabular}

a Data are presented as No.(\%).

Table 2. Mean Score of Perceived Social Support at Different Stages before and after the Intervention ${ }^{\text {a }}$

\begin{tabular}{lcccc}
\hline Time & Before Intervention & $\begin{array}{c}\text { Immediately After } \\
\text { Intervention }\end{array}$ & $\begin{array}{c}\text { Two Months After } \\
\text { Intervention }\end{array}$ & PValue \\
\hline Experimental group & $54.00 \pm 22.1$ & $91.6 \pm 3.6$ & $79.1 \pm 4.1$ & $<0.001$ \\
Control group & $51.3 \pm 10.1$ & - & $50.1 \pm 10.1$ & 0.36 \\
Independent t-test P value & 0.53 & - & $<0.001$ & - \\
\hline
\end{tabular}

a Data are presented as Mean \pm Standard Deviation

\begin{tabular}{lcccc}
\hline Table 3. Mean Score of Self-Care Behaviors at Different Stages before and after the Intervention ${ }^{\mathrm{a}}$ & \\
\hline Time & Before Intervention & $\begin{array}{c}\text { Immediately After } \\
\text { Intervention }\end{array}$ & $\begin{array}{c}\text { Two Months After } \\
\text { Intervention }\end{array}$ & PValue \\
\hline Experimental group & $49.5 \pm 12.3$ & $92.2 \pm 3.2$ & $83.4 \pm 4.7$ & $<0.001$ \\
Control group & $48.9 \pm 6.1$ & - & $48.4 \pm 6.4$ & 0.28 \\
Independent t-test, P value & 0.802 & - & $<0.001$ & - \\
\hline
\end{tabular}

\footnotetext{
a Data are presented as Mean \pm Standard Deviation.
} 


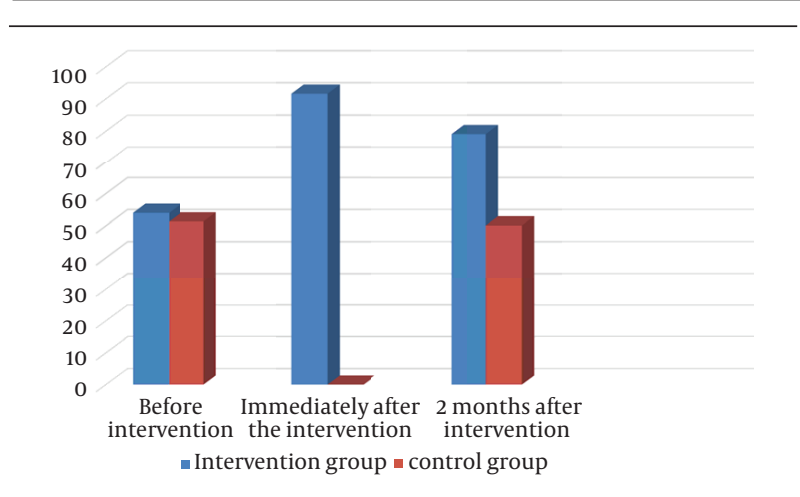

Figure 1. Comparison of the Mean Scores of Perceived Social Support, before, Immediately after, and Two Months after Intervention

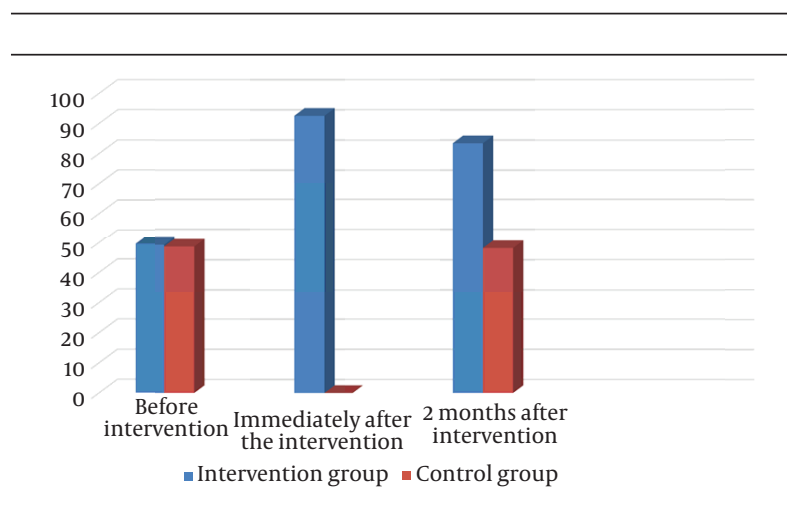

Figure 2. Comparison of the Mean Scores of Self Care, before, Immediately after, and Two Months after Intervention

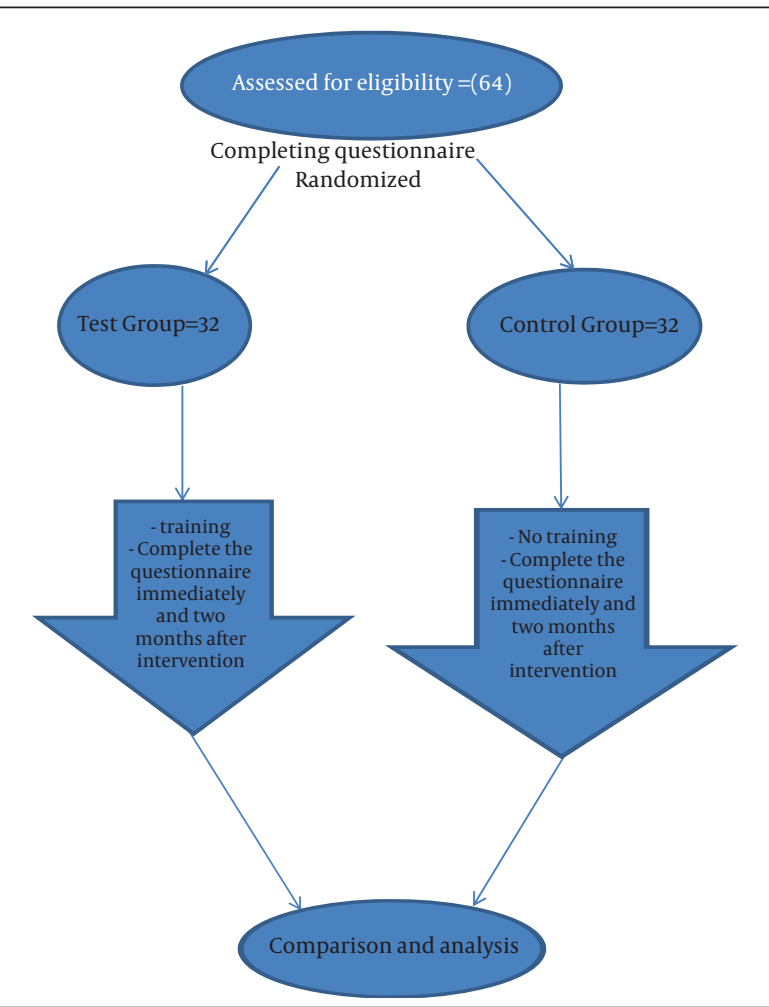

Figure 3. Flow Chart of the Study

\section{Discussion}

The results of this study showed that improvement in perceived social support enhanced self-care behaviors in the heart failure patients in the intervention group. One possible explanation for this finding is that perceived social support can be an effective psychological help to individuals trying to cope with life pressures and issues because they always tend to have this clear notion in their mind that there are people willing to lend them assistance in time of need. Furthermore, perceived social support has a great impact on the level of individual's performance and response to complex problems $(25,26)$. Several studies have shown that social support plays a very important role in the quality of self-care behaviors in chronic patients. The results of the current study were consistent with those from several other studies in this context such as the study of Christensen et al. (21) on 81 patients with renal insufficiency, which demonstrated a strong relationship between perceived social support and self-care behaviors of the patients (e.g. controlling the diet and weighing oneself). However, the authors assessed the impact of perceived social support on self-care behaviors by measuring the serum potassium levels and the weight of their patients, who had renal insufficiency. In the present study, the assessment was performed via a self-reporting questionnaire about self-care behaviors in patients with heart failure. Stanton et al. (27) collected data on 50 patients with hypertension by using a questionnaire and reported a very strong relationship between perceived social support and self-care behaviors. Kulik and Mahler (22) recruited 80 patients with coronary heart disease to investigate the impact of perceived social support on the rate of smoking and walking in these patients. Their results showed that an increase in perceived social support was in tandem with a hike in the walking rate and a drop in the smoking rate. It is noteworthy, however, that whereas the authors focused on only 2 aspects of self-care behaviors, we assessed all facets of practices relating to self-care. Similarly, Wilski et al. (24) also showed that receiving less social support is allied to poorer self-care behaviors among heart failure patients. These results validate the critical role of social support in promoting self-care behaviors. The study of Sullivan and colleagues (12) in Chicago on patients with heart failure showed that social support alleviated depression and anxiety and that an increase in the willingness to perform self-care behaviors was associated with a reduction in re-hospitalization and mortality rates. Along the same lines, Graven et al. (25) also showed that increased levels of social support directly reduced depression and increased self-care behaviors among their study population. Friedman et al. (26) also showed that reducing psychological distress and increasing social support may improve health outcomes among heart failure outpatients. Another study by Farahani and colleagues (20) on patients with heart failure showed a statistically significant 
difference between the effectiveness of the treatment with behavioral activation in reducing anxiety symptoms in the group receiving family support by comparison with a control group within a follow-up period of 6 months $(\mathrm{P}<0.01)$. Another study was conducted on 179 patients with heart failure: in the intervention group, in addition to regular trainings, the patients' relatives were also trained regarding supportive and self-care behaviors. The results showed improved self-care behaviors in both groups; however, this increase was more significant in the test group. In addition, performing self-care behaviors was reduced in both groups after an 8-month followup, with the reduction being more significant in the intervention group (15). Dunbar and colleagues (17) assessed the role of family support in heart failure patients' adherence to a low sodium diet. The authors trained the family members of the patients regarding the diet and found a significant difference between the experimental and control groups. The investigators focused on the performance of only one self-care behavior (low sodium diet) in patients with heart failure, while our study encompassed the entire range of self-care behaviors with respect to the effectiveness of perceived social support among such patients. Our findings showed that the involvement of the patients' family and friends played a major role in the betterment of their self-care behaviors.

\subsection{Limitations}

First and foremost among the limitations in the present study is its small sample size. We would, therefore, recommend future studies with larger sample volumes. The fact that the data were collected through self-reporting tools is another weak point in the current study. It would be advisable that behavioral objective indicators with semi-structured interviews be employed in this field of research. Another weakness of note in our study is the possibility of the existence of unintended adverse trends by the researcher inasmuch as the meetings with the patients and their relatives were held by the researcher. Another salient drawback to our investigation was that the questionnaires were completed by the participants via interviews and, thus, there were some missing values. Nevertheless, it should be noted that the missing values were replaced with mean values.

\section{Acknowledgements}

This article is a part of an MSs thesis approved by the Research Deputyship of Isfahan University of Medical Sciences in 2013 (code \# 392151). We would like to thank the said Deputyship for its support of this study.

\section{Financial Disclosure}

The costs of sample selection and implementation of the training courses given by Isfahan University of Medical Sciences as well as submission fees were paid by Mr. Khaledi.

\section{References}

1. Anderson MA, Levsen J, Dusio ME, Bryant PJ, Brown SM, Burr CM, et al. Evidenced-based factors in readmission of patients with heart failure. J Nurs Care Qual. 2006;21(2):160-7.

2. Heo S, Moser DK, Lennie TA, Riegel B, Chung ML. Gender differences in and factors related to self-care behaviors: a cross-sectional, correlational study of patients with heart failure. Int $J$ Nurs Stud. 2008;45(12):1807-15.

3. Hou N, Chui MA, Eckert GJ, Oldridge NB, Murray MD, Bennett SJ. Relationship of age and sex to health-related quality of life in patients with heart failure. Am J Crit Care. 2004;13(2):153-61.

4. Griffin ML, Amodeo M, Clay C, Fassler I, Ellis MA. Racial differences in social support: kin versus friends. Am J Orthopsychiatry. 2006;76(3):374-80.

5. Konstam MA. Heart failure: evaluation and care of patients with leftventricular systolic dysfunction (Clinical Practice Guidline No. 11).: US Department of Health and Human Services, Public Health Service, Agency for Health Care Policy and Research; 1994.

6. Gerard PS, Peterson LM. Learning needs of cardiac patients. Car dio-Vasc Nurs. 1983;20(2):7-11.

7. Estebsari F, Taghdisi MH, Mostafaei D, Jamshidi E, Latifi M. Determining the factors contributing to quality of life of patients at the last stage of life: a qualitative study. Iran Red Crescent Med J. 2013;15(12):e13594.

8. Waite LJ, Harrison SC. Keeping in touch: How women in midlife allocate social contacts among kith and kin. Social Forces. 1992;70(3):637-54.

9. Greenwood DC, Muir KR, Packham CJ, Madeley RJ. Coronary heart disease: a review of the role of psychosocial stress and social support. J Public Health Med.1996;18(2):221-31.

10. Krumholz HM, Parent EM, Tu N, Vaccarino V, Wang Y, Radford MJ, et al. Readmission after hospitalization for congestive heart failure among Medicare beneficiaries. Arc Intern Med. 1997;157(1):99-104.

11. Felker GM, Kirkwood FA, Wendy AG. Anemia as a Risk Factor and Therapeutic Target in Heart Failure . Heart lung. 2009;12(17):123-8.

12. Sullivan MJ, Wood L, Terry J, Brantley J, Charles A, McGee V, et al The Support, Education, and Research in Chronic Heart Failure Study (SEARCH): a mindfulness-based psychoeducational intervention improves depression and clinical symptoms in patients with chronic heart failure. Am Heart J. 2009;157(1):84-90.

13. Luttik ML, Jaarsma T, Moser D, Sanderman R, van Veldhuisen DJ The importance and impact of social support on outcomes in patients with heart failure: an overview of the literature.J Cardiovasc Nurs. 2005;20(3):162-9.

14. Harrison MB, Browne GB, Roberts J, Tugwell P, Gafni A, Graham ID. Quality of life of individuals with heart failure: a randomized trial of the effectiveness of two models of hospital-to-home transition. Med Care. 2002;40(4):271-82.

15. McAlister FA, Stewart S. Effects of educational and support on self care and resource utilization in patient with heart failure. Eur Health J. 2011;20:673-82.

16. Hope CJ, Wu J, Tu W, Young J, Murray MD. Association of medication adherence, knowledge, and skills with emergency department visits by adults 50 years or older with congestive heart failure. Am J Health Syst Pharm. 2004;61(19):2043-9.

17. Dunbar SB, Clark PC, Deaton C, Smith AL, De AK, O'Brien MC. Family education and support interventions in heart failure: a pilot study. Nurs Res. 2005;54(3):158-66.

18. Artinian NT, Magnan M, Sloan M, Lange MP. Self-care behaviors among patients with heart failure. Heart Lung. 2002;31(3):161-72.

19. Sayers SL. Barbara Riagel DNSC, Social support and self-care of patient with heart failure. Ann Cardiol Angeiol. 2008;35:70-9.

20. Ghaem-magham Farahani Z, Rahimian Boogar I, Najafi M, Forooeddin Adl A, Dabiri S. [The comparison of group behavioural activation with and without familial support effectiveness on anxiety reduction in coronary heart disease]. Heart Lung. 2013;10(2):79-89.

21. Christensen AJ, Smith TW, Turner CW, Holman JM, Jr., Gregory MC, Rich MA. Family support, physical impairment, and adherence in hemodialysis: an investigation of main and buffering effects. J Behav Med. 1992;15(4):313-25. 
22. Kulik JA, Mahler HI. Emotional support as a moderator of adjustment and compliance after coronary artery bypass surgery: a longitudinal study. J Behav Med.1993;16(1):45-63.

23. Sorensen EA, Wang F. Social support, depression, functional status, and gender differences in older adults undergoing first-time coronary artery bypass graft surgery. Heart Lung. 2009;38(4):306-17.

24. Wilski M, Sienkiewicz Wilowska JA. Social support as a regulator of self-care attitude in persons with myocardial infarction. Polish Psychol Bull. 2014;45(4):521-32.

25. Graven LJ, Grant JS, Vance DE, Pryor ER, Grubbs L, Karioth S. The Influence of Social Support and Social Problem-Solving on Depressive Symptomatology and Self-care Behaviors in Individuals with Heart Failure: A Structural Equation Modeling Study. J Card Fail. 2014;20(8):S5.
26. Friedmann E, Son H, Thomas SA, Chapa DW, Lee HJ, Sudden Cardiac Death in Heart Failure Trial I. Poor social support is associated with increases in depression but not anxiety over 2 years in heart failure outpatients. J Cardiovasc Nurs. 2014;29(1):20-8.

27. Stanton AL. Determinants of adherence to medical regimens by hypertensive patients.J Behav Med. 2007;10(4):377-94.

28. Zimet GD, Canty-Mitchell J. Psychometric properties of the Multidimensional Scale of Perceived Social Support in urban adolescents. Am J community Psychol. 2000;28(3):391-400.

29. Davari Dolatabadi E, editor. [Designation of special tools for CHF patient].; 17th Congress of Iranian Heart Association..2011; Tehran.

30. Shojahfard A, Nederyan H. [Effectiveness education on self care and the barriers and bone fits in heart failure patient]. Payavard salamat. 2008;4:43-55. 